\title{
Description and management of non-metastatic thoracic myxofibrosarcoma: a case report
}

\author{
Amir Hossein Aalami ${ }^{1}$, Amir Amirabadii ${ }^{2,3^{*}}{ }^{\mathbb{D}}$, Fatemeh Hosseini Mojahed ${ }^{4}{ }^{\mathbb{D}}$, Ali Ayatollahi ${ }^{(\mathbb{}}$ \\ 'Department of Biology, Mashhad Branch, Islamic Azad University, Mashhad, Iran. \\ ${ }^{2}$ Solid Tumors Research Center, Mashhad University of Medical Sciences, Mashhad, Iran. \\ ${ }^{3}$ Reza Radiation Oncology Center, Mashhad, Iran. \\ ${ }^{4}$ Department of Medical Sciences, Mashhad Medical Sciences Branch, Islamic Azad University, Mashhad, Iran. \\ ${ }^{5}$ Department of Pathology, Mashhad University of Medical Sciences, Mashhad, Iran.
}

*Corresponding Author: Amir Amirabadi; Solid Tumors Research Center, Mashhad University of Medical Sciences, Mashhad, Iran, and Reza Radiation Oncology Center, Mashhad, Iran; Tel: 09155177786, Email: amirabadi1980@yahoo.com

\begin{abstract}
Myxofibrosarcoma is a soft tissue sarcoma (STS) prevailing in the elderly and is associated with metastasis and mortality. In this case, an 88-year-old male is presented with a progressively enlarging mass in the posterior thorax. The physical examination revealed a solid, irregular, painful mass on palpation. Doppler ultrasonography and high-resolution computed tomography (CT) scan revealed a $60 \times 38 \mathrm{~mm}$ hypoechoic tumor in the left paraspinal thoracic area with a little vascularity with no adherence and invasion. Radical excisional surgery was performed. The histopathology findings were in line with myxofibrosarcoma except for CD34. He underwent high dose radiotherapy due to the reported not assessable margins at one side. The patient was asymptomatic and recurrent free in the 12 months follow up assessment. It was the first known case of the non-metastatic intermediate grade of myxofibrosarcoma in the thorax. Due to its deceptive findings, myxofibrosarcoma should always be considered in the assessment of chest wall tumors.

Keywords: Myxofibrosarcoma, Thorax, Surgical resection, Soft tissue neoplasms, Radiation therapy
\end{abstract}

Received: 1 January 2020, Accepted: 8 February 2020, ePublished: 29 June 2020

\section{Introduction}

Myxofibrosarcoma is a kind of soft tissue sarcoma (STS) that one of the most common neoplasms of the limbs of elderly patients reported in the $1970^{\text {th }}(1)$. Although both genders are involved, the prevalence of myxofibrosarcoma among males is higher than females. Myxofibrosarcoma is considered as a common neoplasm of dermal and subcutaneous tissues (1). The signs of myxofibrosarcoma depend on painless mass, slow-growing, skin-colored, or erythematous nodules or tumors (1). Prognostic determinants for this cancer and metastasis-free survival depend on the tumor mass at surgery, histological grade, positive surgical resection margins, percentage of necrosis, and mitotic rate (2). In this case report, an 88-year-old man is presented with a progressively enlarging mass in the posterior thorax without metastasis, and the histological grade was moderately differentiated. The radical surgery and adjuvant Radiotherapy were performed and maintained good health after 12 months follow up.

\section{Case Presentation}

The patient was an 88-year-old man referred to Reza radiotherapy and oncology center in December 2018, with a progressive swelling in the scapula. He reported no salient sickness in his recent medical history. He had Alzheimer's disease for four years before admission and took memantine and donepezil for his condition. The laboratory tests had not remarkable abnormality regarding hormones, urinalysis, and biochemistry except low fasting blood sugar, alkaline phosphatase, and electrolyte disturbances (Table 1). He did not report any significant weight loss. The tumor was illustrated in July 2018 by the patient, which progressively increased in size, reaching the size of an apple (based on the patient's words). The physical examination revealed a large mass in the posterior thoracic area between the scapulas. The tumor was painful in palpation with irregular borders and no mobility. The vital signs of the patient were normal. He did not report smoking or alcohol consumption in the past. He had prostate surgery 20 years ago due to benign prostatic hyperplasia $(\mathrm{BPH})$ and reported a positive family history for cancer (cervical cancer in his mother in the eighth decade of her life). Based on the Eastern Cooperative Oncology Group (ECOG) scoring, he was categorized

C 2020 The Author(s); Published by Shahrekord University of Medical Sciences. This is an open-access article distributed under the terms of the Creative Commons Attribution License (http://creativecommons.org/licenses/by/4.0), which permits unrestricted use, distribution, and reproduction in any medium, provided the original work is properly cited. 
Table 1. The First Patient's Lab Test Report on July 27, 2018

\begin{tabular}{lcc}
\hline Test Name & Result & Reference Range \\
\hline Biochemistry & & \\
\hline Fasting blood sugar (FBS) & $67^{*}$ & $70-105 \mathrm{mg} / \mathrm{dL}$ \\
Cholesterol & 164 & Desirable risk: $<200 \mathrm{mg} / \mathrm{dL}$ \\
HDL & 49 & Men: Low Risk $>50 \mathrm{mg} / \mathrm{dL}$ \\
LDL & 102 & Desirable risk $<100 \mathrm{mg} / \mathrm{dL}$ \\
Triglyceride & 49 & Normal: up to $150 \mathrm{mg} / \mathrm{dL}$ \\
SGOT (AST) & 19 & Up to $40 \mathrm{U} / \mathrm{L}$ \\
SGPT (ALT) & 10 & Up to $40 \mathrm{U} / \mathrm{L}$ \\
Alkaline phosphatase & $283^{*}$ & Men: up to $270 \mathrm{U} / \mathrm{L}$ \\
Na ${ }^{+}$(sodium) & $154^{*}$ & $135-145 \mathrm{mEq} / \mathrm{L}$ \\
K$^{+}$(potassium) & $5.0^{*}$ & $>7$ years old: $3.6-4.8 \mathrm{mEq} / \mathrm{L}$ \\
P (phosphate) & 2.7 & Adults: $2.5-4.7 \mathrm{mg} / \mathrm{dL}$ \\
Ca & 10.1 & $8.6-10.3 \mathrm{md} / \mathrm{dl}$ \\
\hline Hormones \& Vitamins & & \\
\hline T.S.H & 0.882 & $0.3-4.5 \mathrm{mlU} / \mathrm{l}$ \\
T3 & 138.9 & $69-215 \mathrm{ng} / \mathrm{dL}$ \\
PSA & 0.95 & Up to $4 \mathrm{ng} / \mathrm{mL}$ \\
HBS-Ag & Positive $(+)$ & - \\
HBS-Ab & $7.61(-)$ & Negative: $<9 \mathrm{unit} / \mathrm{mL}$ \\
Anti-HCV & Negative (-) & - \\
HIV (1\&2) & Negative (-) & - \\
Vitamin D & \\
Vitamin B12 & 53.1 & Sufficiency: $30-150 \mathrm{ng} / \mathrm{mL}$ \\
\hline Serology & 498 & $191-663 \mathrm{pq} / \mathrm{mL}$ \\
\hline PPD skin test (TB) & Negative (-) & \\
\hline & & \\
\hline
\end{tabular}

in the "symptomatic, $<50 \%$ in bed during the day" status. His body mass index was $16.4 \mathrm{~kg} / \mathrm{m}^{2}$ (weight $=42 \mathrm{~kg}$, height $=160 \mathrm{~cm}$ ). The Color doppler ultrasonography and computed tomography (CT) scan revealed a $60 \times 38 \mathrm{~mm}$ hypoechoic tumor in the left paraspinal thoracic area with no adherence and invasion (Figure 1).

The gross analysis expressed two irregular tan and brown fragments of soft to elastic tissue with marked myxoid changes measuring $8 \times 8 \times 4 \mathrm{~cm}$ in aggregate. The microscopic examination reported a malignant spindle cell neoplasm composed of moderately atypical, pleomorphic cells with a few giant tumor cells, $1 \mathrm{MF} / 10 \mathrm{hpf}$, and fascicular or reticular arrangement with extensive myxoid changes and areas of necrosis and hemorrhage (Figure 2). The immunohistochemistry (IHC) findings in myxofibrosarcoma cells have shown the negative SMA, S-100, desmin, and CD34 but strongly positive for vimentin (Figure 3). Based on the histopathological features and IHC results, the tumor was diagnosed as myxofibrosarcoma. The International Classification of Diseases for Oncology (ICD-O) code determined as C:493 $\mathrm{M}: 8811 / 32$. After surgery, he underwent radiation therapy as adjuvant therapy. Due to the unclear surgical margin on one side at the pathological report, treatment at maximum radiation dose on $3 \mathrm{~d}$ conformal radiation therapy by a high energy linear accelerator machine (LINAC) was chosen. The radiotherapy protocol was a total dose of 66 grays in 33 sessions. After 12 months of follow up, he had no significant side effects and did not show any local recurrence (LR) or distant metastasis (DM) by spiral highresolution CT scan.

\section{Discussion}

Myxofibrosarcoma is recognized as the usual common malignant mesenchymal neoplasm in elderly patients, with slight male predominance. It regularly presents as painless, slow-growing, skin-colored, or erythematous nodules or tumors. Most lesions are positioned in the lower limbs and infrequently on the trunk, head, and neck, with a high rate of LR of $50 \%-60 \%(3,4)$. The recurrence of

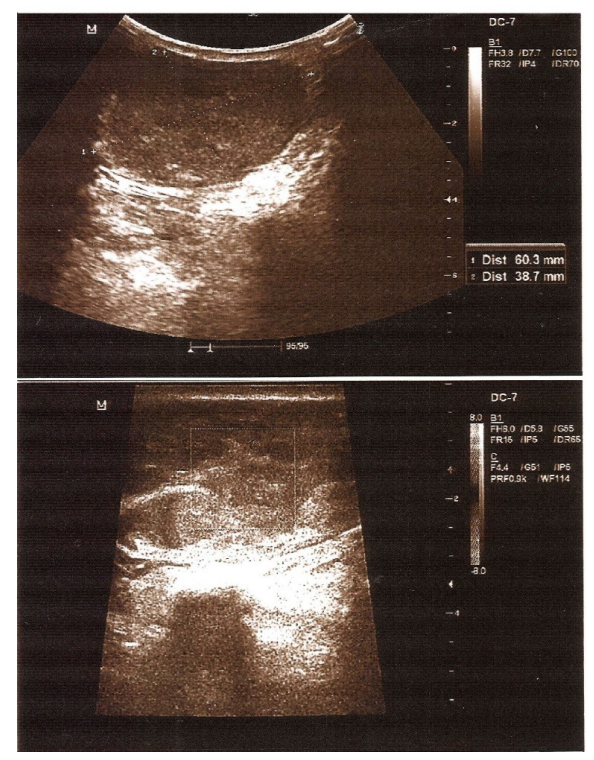

Figure 1. Doppler ultrasonography Imaging assessments revealed a $60 \times 38 \mathrm{~mm}$ hypoechoic tumor in the left paraspinal thoracic area with a little vascularity with no adherence and invasion.

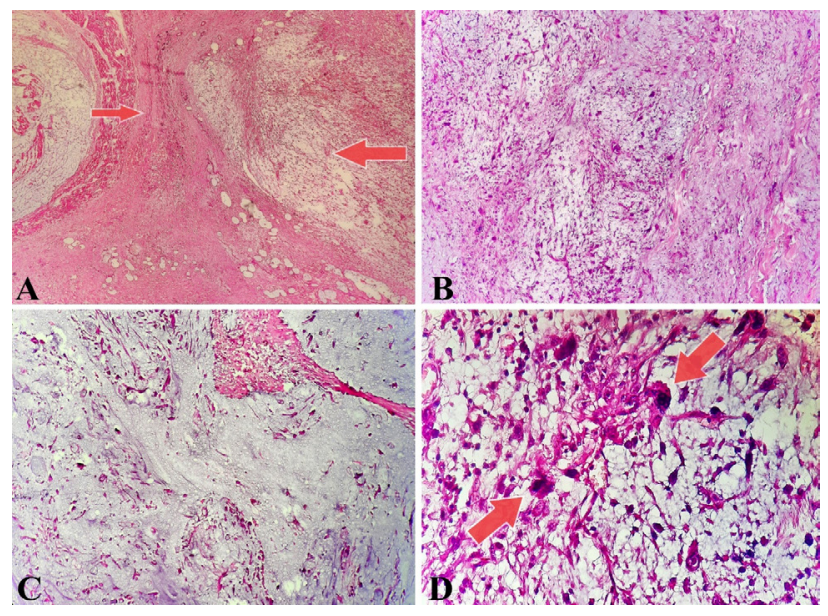

Figure 2. Histopathological images with hematoxylin and eosin stain. A: Two-component of myxofibrosarcoma, myxoid area (thin arrow), the fascicular or reticular arrangement with extensive myxoid changes and areas of necrosis and hemorrhage and cellular area (thick arrow). $(\times 40)$. B: cellular component $(\times 100)$, C: myxoid component $(\times 100)$, D: The atypical cells in the cellular area of myxofibrosarcoma, a malignant spindle cell neoplasm composed of moderately atypical, pleomorphic cells with a few giant tumor cells $(\times 400)$. 


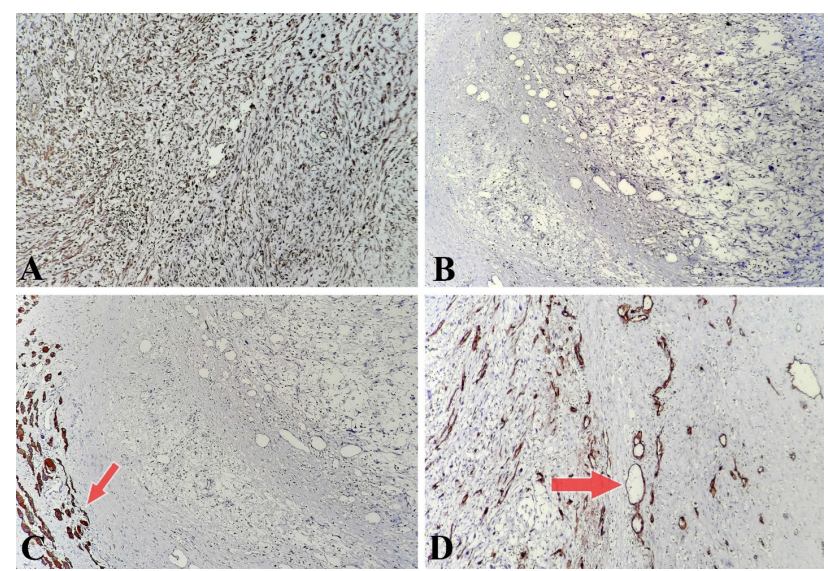

Figure 3. Immunohistochemical reactivity of the tumor cells. Strongly positive for vimentin (A); However, negative for S100 (B), desmin (C), and CD-34 (D). As much as possible, internal control was seen for desmin, and CD34 staining was detected in vascular endothelial cells rather than in tumor cells, highlighting the curvilinear capillaries.

well-differentiated myxofibrosarcoma grade happens in 64\% (5). However, distal metastases are significantly more common with high-grade myxofibrosarcoma at a rate of $33 \%(6)$.

The diagnosis of myxofibrosarcoma is histopathological, and tumors are categorized as low, intermediate, and high grade based predominantly on the level of cellularity (and the appearance of a non-myxoid element) $(3,6)$. The first cell is characterized by the presence of hyperchromatic and pleomorphic cells with extensive myxoid areas. Moderate tumors are associated with recurrent cellular nuclear atypia. Finally, poorly differentiated tumors are solid, pleomorphic cells with numerous mitoses, areas of hemorrhage, and necrosis (3).

The histopathological characteristics of low-grade myxofibrosarcoma, which is also called Evans tumor, include hyalinizing spindle cells with giant rosettes. Lowgrade myxofibrosarcoma is a well-defined, slow-growing, painless mass (5). The median mass size is $6 \mathrm{~cm}$ by imaging but may differ from $1 \mathrm{~cm}$ to $18 \mathrm{~cm}$ (5).

Imaging sometimes provides a histologically definitive diagnosis of STS subtypes and helps in the evaluation of local and DM of the lesion. Chest X-ray is valuable as a screening mechanism, although CT of the thorax is more sensitive to detect pulmonary metastasis. Other useful devices, such as positron emission tomography, and magnetic resonance imaging help detect metastasis in patients with poorly differentiated tumors (7).

No etiologic agents have been clearly defined for STS. Genetic factor plays a role in the onset and progression of sarcomas. It is believed that genetic mutation in mesenchymal pluripotent stem cells creates malignant clones that lead to STS formation (7). Mutations in tumor suppressor genes such as p53, RB-1, and oncogenes have also been linked to STS and may also be involved in its prognosis (7). Other risk factors include exposure to dose-dependent radiation, lipidemia, any exposure to chlorophenols, and more. Some hereditary diseases such as retinoblastoma, type 1 neurofibromatosis, and Gardner's syndrome have STS as an element (7).

The clinical signs associated with the diagnosis of STS are non-specific (7). Due to the low specificity of IHC in myxofibrosarcoma, it plays a restricted role in the determination of myxofibrosarcoma, except to exclude other tumor species in the differential diagnosis (6). The muscle markers, including HHF35, and calponin, are positive in myxofibrosarcoma cells (5). Myxofibrosarcoma cells are usually negative for cytokeratin, CD16, CD68, c-kit (CD117), bcl2, ALK, SMA, desmin, S100, AE1/3, EMA, STAT6, HMB-45, MUC4 and positive for CD99, CD34, MDM2, Vimentin, and CDK4 (8).

In this paper, despite other studies $(1,3,8)$ for the first time, immunohistochemical staining was negative for CD34 in this type of cancer after twice repeated.

Fukui et al (8) reported an 81-year-old man with advanced lung cancer that combined with mediastinal myxofibrosarcoma, that radiation therapy was performed with 39 grays for left iliac bone; However, chemotherapy was performed by pembrolizumab as first-line treatment.

Sugiura et al (9) reported a 74-year-old woman who had LR at 10, 19, 23, and 28 months after primary surgery. Radiation therapy at 45 grays for a tumor that was penetrated the main pulmonary artery resulted in failure after the fourth surgery and died 34 months after the first surgery.

This unexpected disease is usually treated with high-dose radiation. According to studies, doses of 63 grays or more are recommended to improve disease-free survival and overall survival (10). However, the same article noted that patients who received $>68$ grays of radiation had significantly higher complications than those who received a lower dose (10). Our study presents a radiotherapy protocol that a total dose of 66 grays in 33 sessions. During treatment, no severe side effects, including skin reactions and other toxic reactions, were observed. Therefore, chemotherapy was prevented because of aging. After 10 months of follow-up, he had no significant adverse events and showed no LR or DM by high-resolution CT.

\section{Conclusion}

This case was the first sample of a myxofibrosarcoma tumor with moderately differentiated without metastasis, and for the first time, a treatment protocol is presented. Due to its deceptive findings, myxofibrosarcoma should always be considered in the assessment of chest wall tumors.

Conflicts of Interests

The authors declare that they have no competing interests.

Authors Contribution

$\mathrm{AHA}$, and AmA, participated in the design of this study. 
FHM carried out the study with $\mathrm{AHA}$, and they collected important background information and drafted the manuscript.

AlA was the pathologist who evaluated, described, and provided the specimen's H\&E and IHC images. All authors read and approved the final manuscript.

Funding/Support

This research received no particular reward from any funding agency in the public, commercial, or not-for-profit sectors.

Acknowledgments

We would like to thank Reza Radiotherapy and Oncology Center (RROC) and Aria Hospital for their cooperation.

\section{Informed Consent}

Informed consent was obtained from the patient for publication of the report.

\section{References}

1. Jevremovic V, Yousuf A, Hussain Z, Abboud A, Chedrawy EG. A rare presentation of myxofibrosarcoma as a Pancoast tumor: a case report. J Med Case Rep. 2017;11(1):61. doi: 10.1186/ s13256-017-1223-5.

2. Look Hong NJ, Hornicek FJ, Raskin KA, Yoon SS, Szymonifka J, Yeap B, et al. Prognostic factors and outcomes of patients with myxofibrosarcoma. Ann Surg Oncol. 2013;20(1):80-6. doi: 10.1245/s10434-012-2572-3.
3. de Castro BA, Piancastelli AC, Meyer RL, Piancastelli PM, Ribeiro CA, Miranda RM. Myxofibrosarcoma - case report. An Bras Dermatol. 2016;91(1):97-9. doi: 10.1590/abd18064841.20163922.

4. Quimby A, Estelle A, Gopinath A, Fernandes R. Myxofibrosarcoma in head and neck: case report of unusually aggressive presentation. J Oral Maxillofac Surg. 2017;75(12):2709.e1-.e12. doi: 10.1016/j.joms.2017.08.015.

5. Liu D. Tumors and Cancers: Skin-Soft Tissue-Bone-Urogenitals. New York: CRC Press; 2017.

6. Hornick JL. Practical Soft Tissue Pathology: A Diagnostic Approach: A Volume in the Pattern Recognition Series. Philadelphia: Elsevier Health Sciences; 2017.

7. Kotilingam D, Lev DC, Lazar AJ, Pollock RE. Staging soft tissue sarcoma: evolution and change. CA Cancer J Clin. 2006;56(5):282-91-5. doi: 10.3322/canjclin.56.5.282.

8. Fukui T, Wakatsuki Y, Matsukura T. Immunotherapy for advanced lung cancer combined with surgery for mediastinal myxofibrosarcoma: a case report. Surg Case Rep. 2019;5(1):37. doi: 10.1186/s40792-019-0596-7.

9. Sugiura $Y$, Fujimoto $H$, Hashizume $T$, Ogura $G$, Nemoto $E$. An entire clinical course of mediastinal myxofibrosarcoma treated by surgery and radiation. Gen Thorac Cardiovasc Surg. 2018;66(12):748-52. doi: 10.1007/s11748-018-0935-9.

10. Gopalratnam K, Rodriguez JA, Woodson KA, Folman R. A case of myxofibrosarcoma in an unusual thoracic location. Case Rep Oncol. 2016;9(1):39-44. doi: 10.1159/000442875. 